\title{
Nonadiabatic quantum chaos in atom optics
}

\author{
S.V. Prants \\ Laboratory of Nonlinear Dynamical Systems, Pacific Oceanological Institute of the Russian Academy \\ of Sciences, Baltiiskaya St., 43, 690041 Vladivostok, Russia
}

\begin{abstract}
Coherent dynamics of atomic matter waves in a standing-wave laser field is studied. In the dressed-state picture, wave packets of ballistic two-level atoms propagate simultaneously in two optical potentials. The probability to make a transition from one potential to another one is maximal when centroids of wave packets cross the field nodes and is given by a simple formula with the single exponent, the Landau-Zener parameter $\kappa$. If $\kappa \gg 1$, the motion is essentially adiabatic. If $\kappa \ll 1$, it is (almost) resonant and periodic. If $\kappa \simeq 1$, atom makes nonadiabatic transitions with a splitting of its wave packet at each node and strong complexification of the wave function as compared to the two other cases. This effect is referred as nonadiabatic quantum chaos. Proliferation of wave packets at $\kappa \simeq 1$ is shown to be connected closely with chaotic center-of-mass motion in the semiclassical theory of point-like atoms with positive values of the maximal Lyapunov exponent. The quantum-classical correspondence established is justified by the fact that the Landau-Zener parameter $\kappa$ specifies the regime of the semiclassical dynamical chaos in the map simulating chaotic center-of-mass motion. Manifestations of nonadiabatic quantum chaos are found in the behavior of the momentum and position probabilities.
\end{abstract}

Keywords: cold atom, matter wave, quantum chaos

\section{Introduction}

The mechanical action of light upon neutral atoms has been comprehensively studied since the pioneer works of Lebedev, Gerlah and Stern, Kapitza and Dirac and Frisch. The light pressure force provides optical cooling and trapping of atoms [1]. In the last two decades, cold atoms in standing-wave optical fields have been used to study quantum chaos. The proposal [2] to study atomic dynamics in a far-detuned modulated standing wave made atomic optics a testing ground for quantum chaos. A number of impressive experiments have been carried out in accordance with this proposal [3, 4, [5]. New possibilities are opened if one works near the atom-field resonance where the interaction between the internal and external atomic degrees of freedom is intense [ [6, 7, []].

Dynamical chaos in classical mechanics is a special kind of random-like motion without any noise and/or random parameters. It is characterized by exponential sensitivity

\footnotetext{
Email address: prants@poi.dvo.ru, tel.007-4232-312602, fax 007-4232-312573 (S.V. Prants)

URL: http://dynalab. poi.dvo.ru (S.V. Prants)

Preprint submitted to Elsevier 
of trajectories in the phase space to small variations in initial conditions and/or control parameters. Such sensitivity does not exist in isolated quantum systems because their evolution is unitary, and there is no well-defined notion of a quantum trajectory. Thus, there is a fundamental problem of emergence of classical dynamical chaos from more profound quantum mechanics which is known as quantum chaos problem and the related problem of quantum-classical correspondence. In a more general context it is a problem of wave chaos. It is clear now that quantum chaos, microwave, optical, and acoustic chaos [9, 10, 11, 12] have much in common. The common practice is to construct an analogue for a given wave object in a semiclassical (ray) approximation and study its chaotic properties (if any) by well-known methods of dynamical system theory. Then, it is necessary to solve the corresponding linear wave equation in order to find manifestations of classical chaos in the wave-field evolution in the same range of the control parameters. If one succeeds in that the quantum-classical or the wave-ray correspondence are announced to be established.

In atom optics [13] one quantizes both the atomic internal and translational degrees of freedom. The atom is treated as a wave packet which undergoes deformations in the process of exchange of energy and momentum quanta with a light wave. Quantization of the translation motion provides an entanglement of the internal and external degrees of freedom. Any changes in the form of the wave packet will affect the internal state of the atom and vice versa [14, 15]. The optical field provides a tool to manipulate the atomic matter waves. In atom optics the Schrödinger equation for the probability amplitudes constitutes a linear infinite-dimensional dynamical system which is governed by an external force if the field is treated as a classical wave.

In the semiclassical approximation, atom with quantized internal dynamics is treated as a point-like particle with the Hamilton-Schrödinger equations of motion constituting a nonlinear dynamical system. A number of nonlinear Hamiltonian and dissipative dynamical effects have been found with such a system including chaotic Rabi oscillations, chaotic atomic transport, dynamical fractals, synchronization, chaotic walking, and Lévy flights [16, 17, 18, 19, 20, 21]. Similar and new effects have been found numerically and described analytically with two-level atoms in a losseless cavity with a single quantized mode in the framework of the Jaynes-Cummings model [22, 23, 24, 25] and the TavisCummings model 26]. It has been shown that the coupled atom-field dynamics in a cavity can be unstable under appropriate conditions in the absence of any kind of interaction with environment. This kind of quantum instability manifests itself in fractal chaotic scattering of atoms 22, 23, 24, 25], in strong variations of reduced quantum purity and entropy [24, 25, 26], correlating with the respective maximal Lyapunov exponent, and in exponential sensitivity of fidelity of quantum states to small variations of the detuning [24, 25].

The main aim of this paper is to establish a kind of the quantum-classical correspondence in transport properties of point-like atoms and atomic matter waves moving in a standing-waves field. Is the coherent evolution of the atomic matter waves really complicated in that range of the control parameters where the corresponding center-of-mass motion has been shown to be chaotic? 


\section{Wave-packet motion in a standing light wave}

The Hamiltonian of a two-level atom, moving along a one-dimensional classical standingwave laser field, can be written in the frame rotating with the laser frequency $\omega_{f}$ as follows:

$$
\hat{H}=\frac{\hat{P}^{2}}{2 m_{a}}+\frac{\hbar}{2}\left(\omega_{a}-\omega_{f}\right) \hat{\sigma}_{z}-\hbar \Omega\left(\hat{\sigma}_{-}+\hat{\sigma}_{+}\right) \cos k_{f} \hat{X}
$$

where $\hat{\sigma}_{ \pm, z}$ are the Pauli operators for the internal atomic degrees of freedom, $\hat{X}$ and $\hat{P}$ are the atomic position and momentum operators, $\omega_{a}$ and $\Omega$ are the atomic transition and Rabi frequencies, respectively. We will work in the momentum representation and expand the state vector as follows:

$$
|\Psi(t)\rangle=\int[a(P, t)|2\rangle+b(P, t)|1\rangle]|P\rangle d P,
$$

where $a(P, t)$ and $b(P, t)$ are the probability amplitudes to find atom at time $t$ with the momentum $P$ in the excited, $|2\rangle$, and ground, $|1\rangle$, states, respectively. After some algebra one gets the normalized Schrödinger equation for the probability amplitudes [14]

$$
\begin{aligned}
i \dot{a}(p) & =\frac{1}{2}\left(\omega_{r} p^{2}-\Delta\right) a(p)-\frac{1}{2}[b(p+1)+b(p-1)], \\
i \dot{b}(p) & =\frac{1}{2}\left(\omega_{r} p^{2}+\Delta\right) b(p)-\frac{1}{2}[a(p+1)+a(p-1)],
\end{aligned}
$$

where the dot denotes differentiation with respect to dimensionless time $\tau \equiv \Omega t, p \equiv$ $P / \hbar k_{f}$, and $x \equiv k_{f} X$. The normalized recoil frequency $\omega_{r} \equiv \hbar k_{f}^{2} / m_{a} \Omega$ and the atomfield detuning $\Delta \equiv\left(\omega_{f}-\omega_{a}\right) / \Omega$ are the control parameters.

The probability to find an atom with the momentum $p$ at the moment of time $\tau$ is $\mathcal{P}(p, \tau)=|a(p, \tau)|^{2}+|b(p, \tau)|^{2}$. The internal atomic state is described by the following real-valued combinations of the probability amplitudes: $u_{q}(\tau) \equiv 2 \operatorname{Re} \int d p\left[a(p, \tau) b^{*}(p, \tau)\right]$, $v_{q}(\tau) \equiv-2 \operatorname{Im} \int d p\left[a(p, \tau) b^{*}(p, \tau)\right], z_{q}(\tau) \equiv \int d p\left[|a(p, \tau)|^{2}-|b(p, \tau)|^{2}\right]$, which are expected values of the synchronized (with the laser field) and a quadrature components of the atomic electric dipole moment $\left(u_{q}\right.$ and $v_{q}$, respectively) and the atomic population inversion, $z_{q}$. Varying the value of the Rabi frequency $\Omega$, we can change the value of the dimensionless recoil frequency $\omega_{r}$ with the same atom. Working, say, with a cesium atom ( $m_{a}=133$ a.u., $\lambda_{f}=852.1 \mathrm{~nm}$, and $\left.\nu_{\mathrm{rec}} \simeq 2 \mathrm{KHz}\right)$, we get $\omega_{r}=10^{-5}$ at $\Omega=100 \mathrm{MHz}$.

We will interpret the wave-packet motion in the dressed-state basis [13, 27]

$$
|+\rangle_{\Delta}=|2\rangle \sin \Theta+|1\rangle \cos \Theta,|-\rangle_{\Delta}=|2\rangle \cos \Theta-|1\rangle \sin \Theta,
$$

where $\Theta$ is the mixing angle

$$
\tan \Theta \equiv \frac{\Delta}{2 \cos x}-\sqrt{\left(\frac{\Delta}{2 \cos x}\right)^{2}+1} .
$$

These states are eigenstates of an atom at rest in a laser field with the eigenvalues of the quasienergy

$$
E_{\Delta}^{( \pm)}= \pm \sqrt{\frac{\Delta^{2}}{4}+\cos ^{2} x}
$$


The probability amplitudes to find the atom at point $x$ in those potentials are, respectively

$$
C_{+}(x)=a(x) \sin \Theta+b(x) \cos \Theta, C_{-}(x)=a(x) \cos \Theta-b(x) \sin \Theta,
$$

where the amplitudes in the bare-state basis $a(x)$ and $b(x)$ may be computed in the position representation with the help of the Fourier transform

$$
a(x)=\mathrm{const} \int_{-\infty}^{\infty} d p^{\prime} e^{i p^{\prime} x} a\left(p^{\prime}\right), b(x)=\mathrm{const} \int_{-\infty}^{\infty} d p^{\prime} e^{i p^{\prime} x} b\left(p^{\prime}\right) .
$$

Let us assume that we are able to prepare an atom exactly in one of its dressed states, $|+\rangle_{\Delta}$ or $|-\rangle_{\Delta}$. Then the atom will move in one of the potentials, $E_{\Delta}^{(+)}$or $E_{\Delta}^{(-)}$, along a single trajectory. In quantum mechanics, there is a nonzero probability to make a transition to another potential. To estimate this probability we write the Hamiltonian of the internal degree of freedom of a two-level atom in the basis $| \pm\rangle_{\Delta}$

$$
\hat{H}_{\mathrm{int}}=\hat{\sigma}_{z} \cos x+\frac{\Delta}{2} \hat{\sigma}_{x}
$$

Let us linearize the cosine in the vicinity of a node of the standing wave and estimate a small distance the atom makes when crossing the node as follows: $\delta x=\omega_{r}\left|p_{\text {node }}\right| \tau$ [13]. The quantity $\omega_{r}\left|p_{\text {node }}\right|$ is a normalized Doppler shift for an atom moving with the momentum $\left|p_{\text {node }}\right|$, i.e., $\omega_{D} \equiv \omega_{r}\left|p_{\text {node }}\right| \equiv k_{f}\left|v_{\text {node }}\right| / \Omega$. The Schrödinger equation for the probability amplitudes $C_{ \pm}(x)$ in the position representation can be written in the form of the second-order equation

$$
\ddot{C}_{+}(x)+\left[i \omega_{D}+\frac{\Delta^{2}}{4}+\left(\omega_{D} \tau\right)^{2}\right] C_{+}(x)=0 .
$$

The asymptotic solution of Eq. (10),

$$
P_{L Z}=\exp (-\kappa)
$$

gives the probability to make a nonadiabatic or Landau-Zener transition from one of the nonresonant potentials to another one specified by the Landau-Zener parameter

$$
\kappa \equiv \pi \frac{\Delta^{2}}{\omega_{D}}
$$

There are three regimes of atomic motion.

1. $\kappa \gg 1$. The probability to make the transition is exponentially small even when an atom crosses a node. The evolution of the atomic wave packet is adiabatic in this case.

2. $\kappa \ll 1$. The distance between the potentials at the nodes is small and the atom changes the potential each time when crossing any node with the probability close to unity. In the limit case $\Delta=0$, the atom moves in the resonant potentials.

3. $\kappa \simeq 1$. The probability to change the potential or to remain in the same one, upon crossing a node, are of the same order. In this regime one may expect a proliferation of components of the atomic wave packet at the nodes and complexification of the wave function. 


\section{Simulation of ballistic wave-packet propagation}

We simulate the evolution of a Gaussian wave packet with the variance in the momentum space, $\sigma_{p}^{2}=50, p_{0}=10^{3}, x_{0}=0$ and $\omega_{r}=10^{-5}$. The initial average kinetic energy, $\omega_{r} p^{2} / 2=5$, is greater than the depth of the potential wells, so the atom will move ballistically along the positive direction of the standing-wave axis.

To study all the regimes of the wave-packet motion, we simulate Eqs. (3) at different values of the Landau-Zener parameter $\kappa$ (12). The normalized Doppler shift $\omega_{D}$ nearby a node of the standing wave is estimated to be $\omega_{D} \simeq \omega_{r} p_{0}=0.01$. If we choose, say, $\Delta=0.3$ we get the first case in our nomenclature, $\kappa \gg 1$, with exponentially small probability of nonadiabatic transitions. The wave packet, initially prepared in the ground state which is a superposition of the dressed states with approximately equal weights, splits from the beginning (Fig. 17) into two components, $|+\rangle_{\Delta}$ and $|-\rangle_{\Delta}$, each of which moves in its own nonresonant potential, $E_{\Delta}^{(+)}$or $E_{\Delta}^{(-)}$. We really do not observe in Fig. 17a any splitting at the nodes, and the motion of the wave packet at $\Delta=0.3$ is adiabatic and practically periodic. If $\kappa \ll 1$ (the motion near the resonance), one expects to observe the periodic motion in the two resonant potentials simultaneously without any splitting 14.

At $\Delta=0.1$, we get $\kappa \simeq 1$ and expect nonadiabatic transitions at the nodes of the standing wave in accordance with formula (11). The initial ground state $|1\rangle$ is now a superposition of the dressed states with practically the same weights. The initial bifurcation is accompanied by splittings (see Fig. 10) that can be proved to occur at the nodes of the standing wave. Let us start to analyze the wave-packet motion with the $|+\rangle_{\Delta}$-component (the upper curve in the figure). The first splitting occurs at $\tau_{1}^{(+)} \simeq 150$. It is easy to prove that it is the moment of time when the centroid of the $|+\rangle_{\Delta}$-component crosses the first node at $x=\pi / 2: \tau_{1}^{(+)}=\pi / 2 \omega_{r} \bar{p}_{0,1}^{(+)} \simeq 150$, where $\bar{p}_{0,1}^{(+)}$is the average momentum of the centroid of the $|+\rangle_{\Delta}$ wave packet between $x=0$ and $x=\pi / 2$. Thus, the wave packet, crossing the node, splits into two parts. The first one prolongs its motion in the potential $E_{\Delta}^{(+)}$after passing the point $x=\pi / 2$. It is the lower curve in Fig. 1b starting at $\tau_{1}^{(+)} \simeq 150$. The corresponding packet slows down because this component loses its kinetic energy going up to the top of the potential $E_{\Delta}^{(+)}$. As to the second trajectory (the upper curve starting at $\tau_{1}^{(+)}$), it appears due to the nonadiabatic transition to the potential $E_{\Delta}^{(-)}$. That is why it accelerates from the beginning and reaches its maximal velocity at $x=\pi$. The $|-\rangle_{\Delta}$-component (the lower curve starting at $\tau=0$ ) splits at the first node at $\tau_{1}^{(-)}=\pi / 2 \omega_{r} \bar{p}_{0,1}^{(-)} \simeq 156$. In course of time both the components split at every node of the standing wave at the moments $\tau_{n}^{( \pm)}$that can be estimated with the simple formula

$$
\omega_{r} \bar{p}_{n-1, n}^{( \pm)} \tau_{n}^{( \pm)}=(2 n-1) \frac{\pi}{2}, n=2,3, \ldots,
$$

where $\bar{p}_{n-1, n}^{( \pm)}$is an average momentum of the centroid of the corresponding component between the $(n-1)$-th and $n$-th nodes. Such a proliferation at the nodes means a complexification of the atomic wave function both in the momentum and position spaces as compared to the adiabatic and resonant cases.

Now we go to the position space and compute the probability $|C(x, \tau)|^{2}=\left|C_{-}(x, \tau)\right|^{2}+$ $\left|C_{+}(x, \tau)\right|^{2}$ to be at point $x$ at time $\tau$. In Fig. 2 we show the result of simulation in the 
case of adiabatic and nonadiabatic motion at $\Delta=0.3$ and $\Delta=0.1$ corresponding, respectively, to Fig. 1 $\mathrm{a}$ and $\mathrm{b}$ in the momentum space. It is a plot of the position probability in the frame moving with the initial atomic velocity $\omega_{r} p_{0}=0.01$ where the slope straight lines mark positions of the nodes of the standing wave in the moving frame. At $\Delta=0.3$, the evolution is simple without any transitions at the nodes (Fig. 22 ). The splitting of the total probability $|C(x, \tau)|^{2}$ is caused by the initial bifurcation of the wave packet due to its bipotential motion.

The situation is cardinally different when we work in the regime with nonadiabatic transitions at the field nodes $(\Delta=0.1)$. Splitting at the nodes in the momentum space (see Fig 1b) manifest itself in the position space in Fig. 2b. In this case one observes visible changes in the proba6bility $|C(x)|^{2}$ exactly at the node lines. It is a clear evidence of the nonadiabatic transitions that occur in the specific range of the control parameters, $\kappa \simeq 1$. This results in a proliferation of components of the wave packet at the nodes and, therefore, a complexification of the wave function both in the momentum and position spaces.

\section{Quantum-classical correspondence and nonadiabatic quantum chaos}

In this section we compare the quantum results, obtained in the preceding sections, with those obtained for the same problem but in the semiclassical approximation when the translational motion has been treated as a classical one [6, 7, 8, 17, 19]. Coherent semiclassical evolution of a point-like two-level atom is governed by the Hamilton-Schrödinger equations with the same normalization as in the quantum case

$$
\begin{gathered}
\dot{x}=\omega_{r} p, \quad \dot{p}=-u \sin x, \quad \dot{u}=\Delta v, \\
\dot{v}=-\Delta u+2 z \cos x, \quad \dot{z}=-2 v \cos x,
\end{gathered}
$$

where

$$
u \equiv 2 \operatorname{Re}\left(a_{0} b_{0}^{*}\right), v \equiv-2 \operatorname{Im}\left(a_{0} b_{0}^{*}\right), z \equiv\left|a_{0}\right|^{2}-\left|b_{0}\right|^{2}
$$

are the atomic-dipole components ( $u$ and $v$ ) and population-inversion $(z)$, and $a_{0}$ and $b_{0}$ are the complex-valued probability amplitudes to find the atom in the excited and ground states, respectively. The system (14) has two integrals of motion, the total energy

$$
H \equiv \frac{\omega_{r}}{2} p^{2}-u \cos x-\frac{\Delta}{2} z,
$$

and the length of the Bloch vector, $u^{2}+v^{2}+z^{2}=1$.

Equations (14) constitute a nonlinear Hamiltonian autonomous system with two and half degrees of freedom and two integrals of motion. It has been shown in Ref. [19] to have positive values of the maximal Lyapunov exponent $\lambda$ in a wide range of values of the control parameters and initial states. This fact implies dynamical chaos in the sense of exponential sensitivity to small changes in initial conditions and/or control parameters. The result of computation of the maximal Lyapunov exponent in dependence on the detuning $\Delta$ and the initial Doppler shift $\omega_{D}=\omega_{r} p_{0}$ is shown in Fig. 3 at $\omega_{r}=10^{-5}$. In white regions of the plot the values of $\lambda$ are almost zero, and the atomic motion is regular in the corresponding ranges of $\Delta$ and $\omega_{D}$. In shadowed regions positive values of $\lambda$ imply unstable motion. At exact resonance, we get $\lambda=0$ because at $\Delta=0$ the semiclassical 
equations of motion (14) become integrable due to an additional integral of motion, $u=$ const. We stress that the local instability produces chaotic center-of-mass motion in a rigid standing wave without any modulation of its parameters in difference from the situation with atoms in a periodically kicked optical lattice [3, 4, 5]. In dependence on the initial conditions and the parameter values, an atom may oscillate in a well of the lattice or it may have enough kinetic energy to overcome the potential barrier. In some cases the center-of-mass motion resembles a random walking. It means that an atom in a deterministic standing-wave field alternates between flying through the lattice, and being trapped in its wells. Moreover, it may change the direction of motion in a random-like way (see Ref. [17, 19] for coherent Hamiltonian dynamics and Refs. [20, 21] for a dissipative one with spontaneous emission included).

It follows from (14) that the translational motion is described by the equation for a nonlinear physical pendulum with the frequency modulation

$$
\ddot{x}+\omega_{r} u(\tau) \sin x=0,
$$

where $u$ is a function of all the other dynamical variables. It has been shown in Ref. [19] that the regime of the center-of-mass motion is specified by the character of oscillations of the component $u$ of the Bloch vector. In a chaotic regime sudden "jumps" of the variable $u$ occur when an atom crosses the field nodes. Figure 4 a demonstrates more or less periodic oscillations of $u$ at the detuning value $\Delta=0.3$ at which the corresponding maximal Lyapunov exponent is zero (Fig. 3). In the chaotic regime at $\Delta=0.1 u$ demonstrates shallow oscillations interrupted by jumps of different amplitudes upon crossing the nodes (Fig. 4b).

Approximating the variable $u$ between the nodes by constant values, the following stochastic map has been constructed in Ref. [19]

$$
u_{m}=\sin \left(\Theta \sin \varphi_{m}+\arcsin u_{m-1}\right),
$$

where $\Theta \equiv \sqrt{\pi \Delta^{2} / \omega_{r} p_{\text {node }}}$ is an angular amplitude of the jump, $u_{m}$ is a value of $u$ just after the $m$-th node crossing, $\varphi_{m}$ are random phases to be chosen in the range $[0,2 \pi]$, and $p_{\text {node }} \equiv \sqrt{2 H / \omega_{r}}$ is the value of the atomic momentum at the instant when the atom crosses a node (which is the same with a given value of the energy $H$ for all the nodes). With given values of $\Delta, \omega_{r}$ and $p_{\text {node }}$, the map (18) has been shown numerically to give a satisfactory probabilistic distribution of magnitudes of changes in the variable $u$ just after crossing the nodes. The stochastic map (18) is valid under the assumptions of small detunings $(|\Delta| \ll 1)$ and comparatively slow atoms $\left(\left|\omega_{r} p\right| \ll 1\right)$. Furthermore, it is valid only for those ranges of the control parameters and initial conditions where the motion of the basic system (14) is unstable. For example, in those ranges where all the Lyapunov exponents are zero, $u$ becomes a quasi-periodic function and cannot be approximated by the map (see Fig. 目).

The key result in the context of the quantum-classical correspondence is that the squared angular amplitude of the map (18) is exactly the Landau-Zener parameter (12), i.e., $\Theta^{2}=\kappa$. Rewriting the map (18) for $\arcsin u_{m}$, one gets

$$
\arcsin u_{m}=\sqrt{\kappa} \sin \varphi_{m}+\arcsin u_{m-1},
$$

where the jump magnitude does not depend on a current value of the variable. The map (19) visually looks as a random motion of the point along a circle of unit radius (see Fig. 4 
in Ref. [19]). If $\kappa \simeq 1$, then the internal atomic variable $\arcsin u_{m}$ just after crossing the $m$-th node may take with the same probability practically any value from the range $[-\pi / 2, \pi / 2]$. It means semiclassically that the momentum of a ballistic atom changes chaotically upon crossing the field nodes. In accordance with the quantum formula (12), the corresponding atomic wave packet makes nonadiabatic transitions when crossing the nodes and splits at each node (see Figs. 1 $\mathrm{b}$ and $2 \mathrm{~b}$ ). As the result, the wave packet of a single atom becomes so complex that it may be called a chaotic one in the sense that it is much more complicated than the wave packets propagating adiabatically. Thus, nonadiabatic wave chaos and semiclassical dynamical chaos occur in the same range of the control parameters and are specified by the same Landau-Zener parameter $\kappa \simeq 1$. In two limit cases with $\kappa \ll 1$ and $\kappa \gg 1$ both the semiclassical and quantized translational ballistic motion are regular.

In quantum mechanics there is no well-defined notion of a trajectory in the phase space and, hence, the Lyapunov exponents can not be computed. In quantum mechanics there is no exponential sensitivity to small variations in initial conditions because the time evolution of an isolated quantum system is unitary, and the overlap of any two different quantum state vectors is a constant in course of time. Moreover, quantum phase space is discrete due to the Heisenberg uncertainty principle unlike continuous classical phase space. Namely the continuity of the classical phase space provides a possibility of chaotic mixing which exploits more and more fine structures in the classical phase space in course of time whereas the quantum evolution stops to do that over a rather short Ehrenfest time.

The semiclassical (14) and quantum (3) equations of motion look very different. The semiclassical ones constitute a five-dimensional nonlinear dynamical system of ODEs with two integrals of motion that has been shown to be chaotic in a certain range of control parameters with exponential sensitivity to small variations to initial conditions [19]. The quantum ones constitute an infinite-dimensional set of linear equations. It is not evident a priori that their solutions might demonstrate a kind of correspondence in the same range of the control parameters. Nevertheless, a sort of quantum-classical correspondence both in regular and chaotic regimes of the center-of-mass motion has been found. It should be stressed that this correspondence manifests itself in behavior of the quantum Bloch variable $u$ in the semiclassical equations of motion (14).

However, the quantum-classical correspondence is not and could not be absolute because the Planck constant is equal to 1 with our normalization. It cannot tend to be zero in order to achieve a classical limit as it could be done with an effective Planck constant (see, for example, Refs. [2, 5]) depending on the system's parameters. We work in this sense in a deep quantum regime. The quantum-classical dualism with cold atoms resembles the wave-ray one in a classical wave motion. In the context of this paper we might compare ray-like trajectories of atoms with their wave-like motion.

To illustrate correspondence and difference that inavoidably appears when comparing quantum evolution with the classical one (that is only an approximation to the quantum one), we compute with Eqs. (14) the evolution of a Gaussian distribution over classical momentum $p$ and position $x$ with the same parameter's values as in simulation of the wave-packet propagation shown in Figs. 1 and 2, In accordance with the Lyapunov map in Fig. 3, one expects a regular center-of-mass motion at the detuning $\Delta=0.3$ and a weakly chaotic one at $\Delta=0.1$. In Figs. $5 \mathrm{~b}$ and b evolution of classical momenta is shown for the regular $(\Delta=0.3)$ and chaotic $(\Delta=0.1)$ regimes of the center-of-mass 
motion. Visible spreading in $p$ with chaotically moving atoms, as compared to regularly moving ones, is one of the signs of classical dynamical chaos. Figure 1 demonstrates similar spreading of the momentum probability distribution of a Gaussian wave packet with nonadiabatic transitions at $\Delta=0.1$ (Fig. 1b) as compared to the adiabatically moving wave packet at $\Delta=0.3$ (Fig. 1 $\mathrm{k}$ ). The difference between the classical and quantum evolution is also evident: the semiclassical equations of motion (14) are not able to simulate the splitting of wave packets due to purely quantum effect of motion in two optical potentials simultaneously.

In Figs. 6 $\mathrm{b}$ and $\mathrm{b}$ we plot, respectively, regular and chaotic trajectories in the frame of reference moving with the initial atomic velocity $\omega_{r} p_{0}=0.01$. The bundle of chaotically moving atoms in Fig. 6b diverges in a short time significantly as compared to the regular one in Fig. 6a. This property can be used to detect chaotic scattering in a real experiment with atoms crossing a standing laser wave [15]. As to quantum motion in the position space, it is evident that the wave packet with nonadiabatic transitions (Fig. 2b) becomes much broader in course of time due to splitting at the standing-wave nodes than the adiabatic wave packet in Fig. $2 \mathrm{a}$ resembling a broadening of the bundle of chaotic pointlike atoms (Fig. 6b) as compared to the regular one (Fig. 6h). However, we do not observe any splitting of the classical bundles because a classical trajectory simulates only the motion of the centroid of a quantum wave packet and cannot simulate of course its splitting due to purely quantum effect of motion in two optical potentials simultaneously. There is only one optical potential in the semiclassical approximation.

\section{Conclusion}

We have studied coherent dynamics of ballistic atomic wave packets in a one-dimensional standing-wave laser field. The problem has been considered in the momentum representation and in the dressed-state basis where the motion of a two-level atom was interpreted as a motion in two optical potentials. The character of that motion has been shown to depend strongly on the value of the Landau-Zener parameter $\kappa$ (12). If $\kappa \gg 1$, then the probability of transitions from one of the potential to another one, which is described by the Landau-Zener formula (11), is exponentially small. Under such a condition, atoms move in the adiabatic regime. If $\kappa \ll 1$, the formula (11) gives almost unity probability to change the potential when crossing the nodes. In the intermediate case, $\kappa \simeq 1$, the probabilities for an atom to change or not to change the nonresonant potential, when crossing a node, are of the same order. The corresponding nonadiabatic transitions manifest themselves as a splitting of the atomic wave packets in the momentum space when their centroids cross the nodes. This nonadiabatic quantum chaos occurs exactly in the same range of the detuning and the Doppler shift where the semiclassical dynamics has been shown to be chaotic. It is remarkable that the same Landau-Zener parameter $\kappa$ specifies both semiclassical and quantum chaos with ballistic atoms in a deterministic optical lattice.

We hope that the results obtained can be used to study manifestations of quantum chaos with Bose-Einstein condensates in optical lattices [28] with coupled degrees of freedom. From the theoretical point of view, the dynamics of condensates of ultracold atoms is described correctly by the Gross-Pitaevskii equation which is a kind of a nonlinear Schrödinger equation with possible chaotic solutions. Experimentally, one of the possibilities is to prepare two Bose-Einstein condensates in different internal states [29]. 
Another possibility can be realized with a Bose-Einstein condensate in an optical lattice subject to a static tilted force [30, 31, 32]. Viewing transitions between the Bloch bands of a condensate in such a tilted optical lattice as a two-state problem, we get a mesoscopic quantum system with coupled different degrees of freedom (I am thankful to an anonymous referee for that comment).

\section{Acknowledgments}

This work was supported by the Russian Foundation for Basic Research (project no. 09-02-00358), by the Integration grant from the Far-Eastern and Siberian branches of the Russian Academy of Sciences, and by the Program "Fundamental Problems of Nonlinear Dynamics" of the Russian Academy of Sciences. I thank L.E. Konkov and V.O. Vitkovsky for their help in preparing some figures.

\section{References}

[1] V. Letokhov, Laser control of atoms and molecules, Oxford University Press, New York, 2007.

[2] R. Graham, M. Schlautmann, P. Zoller, Dynamical localization of atomic-beam deflection by a modulated standing light wave, Phys. Rev. A 45 (1992) 19-22.

[3] F. L. Moore, J. C. Robinson, C. Bharucha, P. E. Williams, M. G. Raizen, Observation of dynamical localization in atomic momentum transfer: a new testing ground for quantum chaos, Phys. Rev. Lett. 73 (1994) 2974-2977.

[4] D. A. Steck, W. H. Oskay, M. G. Raizen, Observation of chaos-assisted tunneling between islands of stability, Science 293 (2001) 274-278.

[5] W. K. Hensinger, N. R. Heckenberg, G. J. Milburn, H. Rubinsztein-Dunlop, Experimental tests of quantum nonlinear dynamics in atom optics, J. Opt. B: Quantum Semiclass. Opt. 5 (2003) 83-120.

[6] S. V. Prants, L.E. Kon'kov, Chaotic motion of atom in the coherent field of a standing light wave, JETP Letters 73 (2001) 180-183 [Pis'ma ZETP 73 (2001) 200-204].

[7] S.V. Prants, V.Yu. Sirotkin, Effects of the Rabi oscillations on the atomic motion in a standing-wave cavity, Phys. Rev. A 64 (2001) 033412.

[8] S. V. Prants, Chaos, fractals and flights of atoms in cavities, JETP Letters 75 (2002) 651-658 [Pis'ma ZETP 75 (2002) 777-785].

[9] F. Haake, Quantum signatures of chaos, Springer-Verlag, Berlin, 2001.

[10] L. Reichl, The transition to chaos in conservative classical systems: quantum manifestations, Springer-Verlag, New York, 1992.

[11] H.-J. Stöckmann, Quantum Chaos: An Introduction, Cambridge University Press, Cambridge, 1999.

[12] D. Makarov, S. Prants, A. Virovlyansky, G. Zaslavsky, Ray and wave chaos in ocean acoustics: chaos in waveguides, World Scientific, Singapore, 2010.

[13] A. P. Kazantsev, G. I. Surdutovich, V. P. Yakovlev, Mechanical Action of Light on Atoms, World Scientific, Singapore, 1990.

[14] S. V. Prants, Proliferation of atomic wave packets at the nodes of a standing light wave, JETP 109 (2009) 751-761 [ZETP 136 (2009) 872-884].

[15] S. V. Prants, On the possibility of observing nonadiabatic atomic transitions in a laser field and their application to nanolithography, JETP Letters 92 (2010) 726-730 [Pis'ma ZETP 92 (2010) 808-813].

[16] S. V. Prants, M. Edelman, G. M. Zaslavsky, Chaos and flights in the atom-photon interaction in cavity QED, Phys. Rev. E, 66 (2002) 046222.

[17] V. Yu. Argonov, S. V. Prants, Fractals and chaotic scattering of atoms in the field of a stationary standing light wave, JETP 96 (2003) 832-845 [ZETP 123 (2003) 946-961].

[18] V.Yu. Argonov, S.V. Prants, Synchronization of internal and external degrees of freedom of atoms in a standing laser wave, Phys. Rev. A. 71 (2005) 053408.

[19] V. Yu. Argonov, S. V. Prants, Theory of chaotic atomic transport in an optical lattice, Phys. Rev. A 75 (2007) art. 063428. 
[20] V. Yu. Argonov, S. V. Prants, Theory of dissipative chaotic atomic transport in an optical lattice, Phys. Rev. A 78 (2008) 043413.

[21] V. Yu. Argonov, S. V. Prants, Nonlinear control of chaotic walking of atoms in an optical lattice, Europhys. Lett. 81 (2008) 24003.

[22] S.V. Prants, M.Yu. Uleysky. Atomic fractals in cavity quantum electrodynamics, Phys. Lett. A. 309 (2003) 357-362.

[23] M. Uleysky, L. Kon'kov, S. Prants. Quantum chaos and fractals with atoms in cavities, Communications in Nonlinear Science and Numerical Simulation 8 (2003) 329-347.

[24] S.V. Prants, M.Yu. Uleysky, V.Yu. Argonov. Entanglement, fidelity, and quantum-classical correlations with an atom moving in a quantized cavity field, Phys. Rev. A. 73 (2006) art. 023807.

[25] S.V. Prants. Entanglement, fidelity and quantum chaos in cavity QED, Communications in Nonlinear Science and Numerical Simulation. 12 (2007) 19-30.

[26] L. Chotorlishvili, Z. Toklikishvili, S. Wimberger, J. Berakdar, Phys. Rev. A. 84 (2011) art. 013825.

[27] C. Cohen-Tannoudji, J. Dupon-Roc, G. Grynberg, Atom-Photon Interaction, Wiley, Weinheim, 1998.

[28] O. Morsch, M. Oberthaler. Dynamics of Bose-Einstein condensates in optical lattices, Rev. Mod. Phys. 78 (2006) 179-215.

[29] M.R. Matthews, B.P. Anderson, P.C. Haljan, D.S. Hall, C.E. Wieman, E.A. Cornel. Vortices in a Bose-Einstein Condensate, Phys. Rev. Lett. 83 (1999) 2498-2501.

[30] M. Jona-Lasinio, O. Morsch, M. Cristiani, N. Malossi, J. H. Müller, E. Courtade, M. Anderlini, E. Arimondo. Asymmetric Landau-Zener Tunneling in a Periodic Potential, Phys. Rev. Lett. 91 (2003) art. 230406.

[31] A. Kolovsky, H.J. Korsch, E.-M. Graefe. Bloch oscillations of Bose-Einstein Condensates: Quantum counterpart of dynamical instability, Phys. Rev. A. 80 (2009) art. 023617.

[32] G. Tayebirad, A. Zenesini, D. Ciampini, R. Mannella, O. Morsch, E. Arimondo, N. Lörch, S. Wimberger. Time-resolved measurement of Landau-Zener tunneling in different bases, Phys. Rev. A. $82(2010)$ art. 013633.
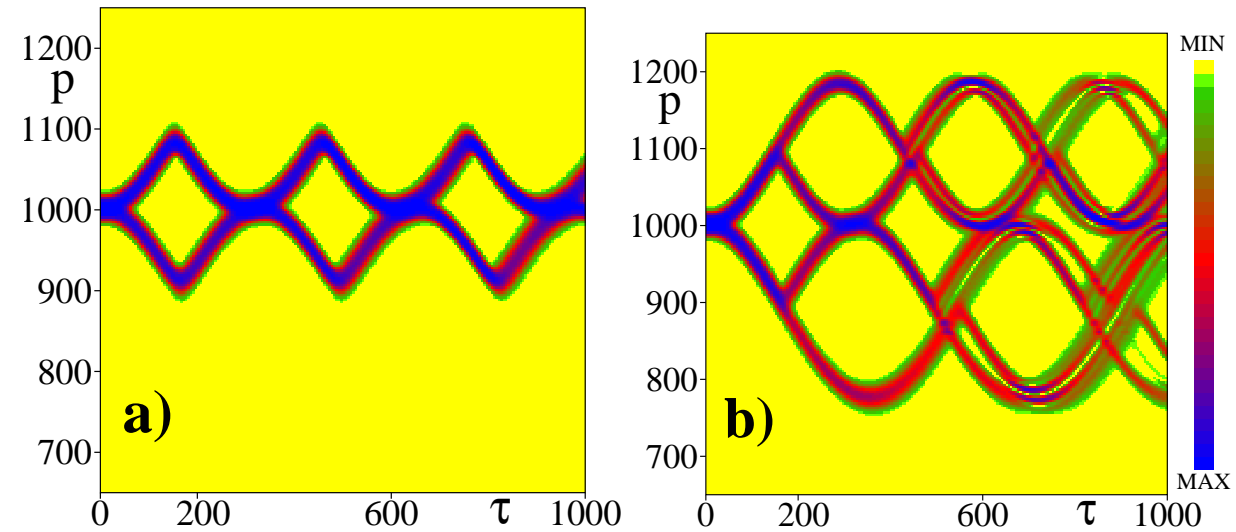

Figure 1: (Color online) Momentum probability distribution $\mathcal{P}(p, \tau)$ of a Gaussian wave packet vs time with $p_{0}=1000, \sigma_{p}^{2}=50$, and $\omega_{r}=10^{-5}$ at (a) $\Delta=0.3$, adiabatic motion, and (b) $\Delta=0.1$, motion with nonadiabatic transitions. The color codes the values of $\mathcal{P}(p, \tau)$. 

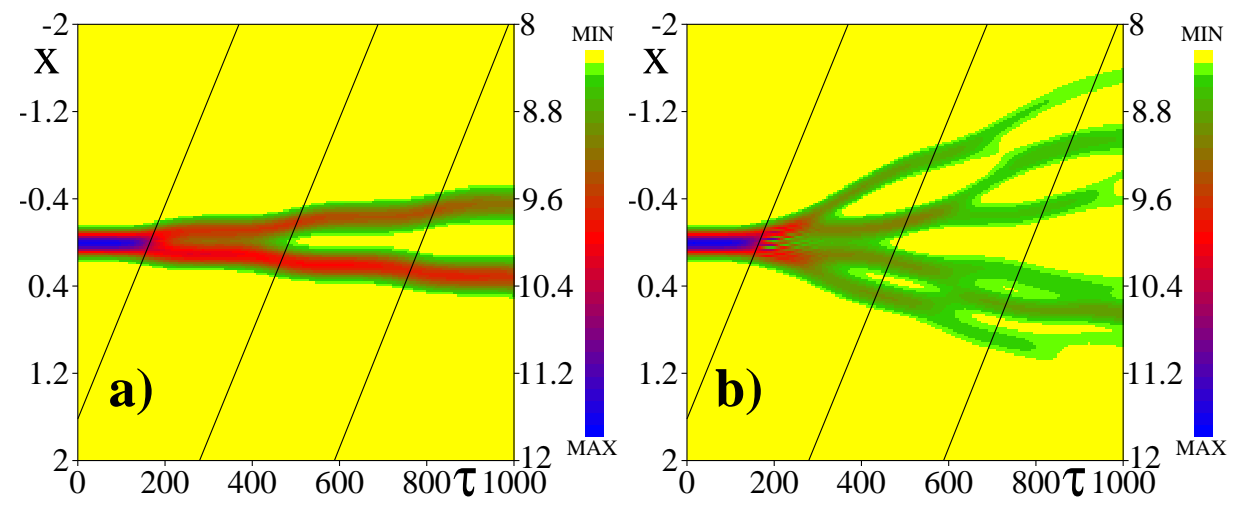

Figure 2: (Color online) The position probability $|C(x)|^{2}$ in the moving frame of reference with the slope straight lines marking positions of the nodes. (a) Adiabatic motion in the position space at $\Delta=0.3$. (b) Wave-packet propagation in the position space with nonadiabatic transitions at the field nodes.

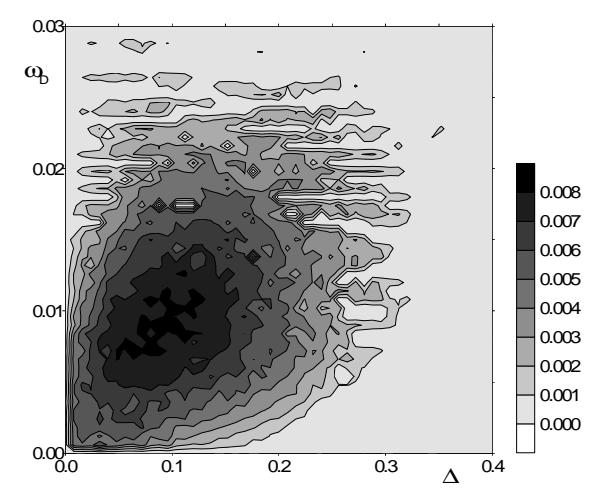

Figure 3: Maximal Lyapunov exponent $\lambda$ vs atom-field detuning $\Delta$ and the initial Doppler shift $\omega_{D}=$ $\omega_{r} p_{0}$. Color codes the values of $\lambda$.
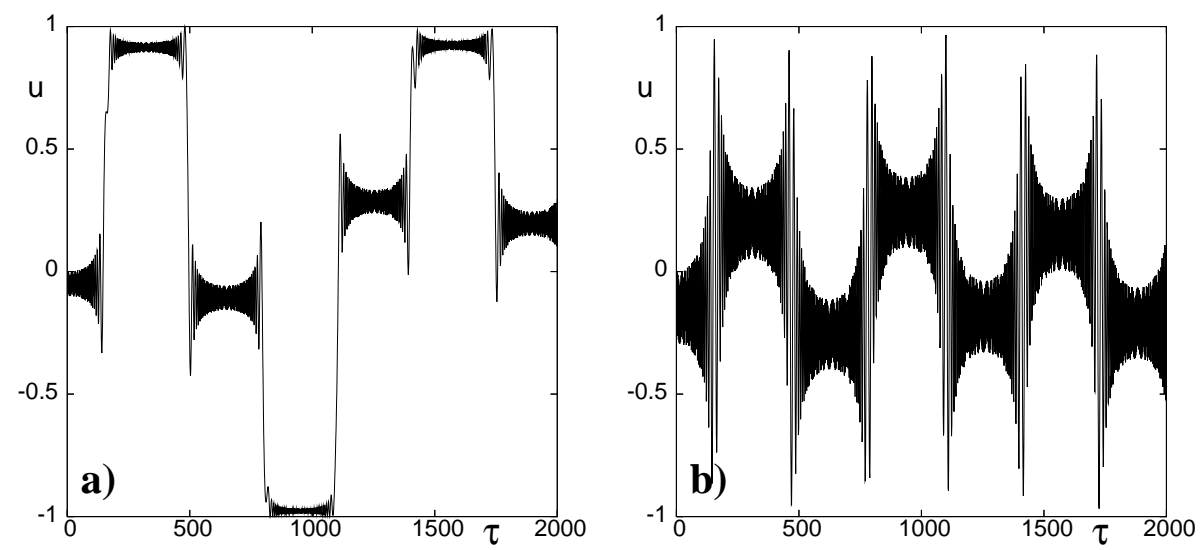

Figure 4: Semiclassical evolution of the atomic-dipole component $u$ in (a) regular $(\Delta=0.3)$ and (b) chaotic $(\Delta=0.1)$ regimes of the ballistic motion of a point-like atom. 

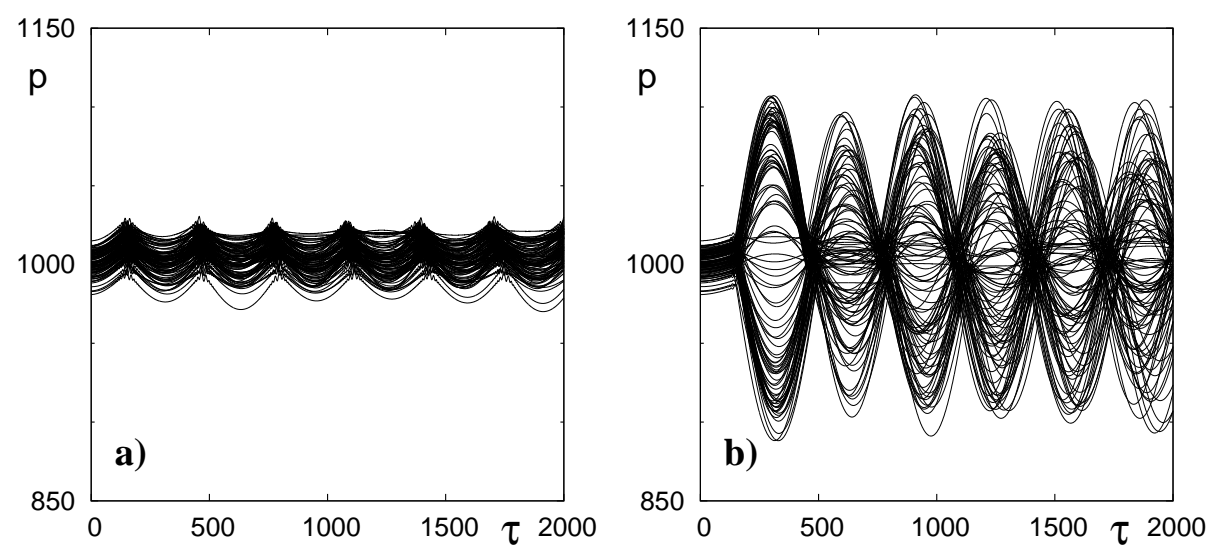

Figure 5: Atomic trajectories in the momentum space computed with the classical Gaussian distribution at the same parameter's values as in simulation with Gaussian wave packets. (a) Regular center-of-mass motion $(\Delta=0.3)$ corresponding to adiabatic quantum motion in Fig. 1 a and (b) weakly chaotic motion $(\Delta=0.1)$ corresponding to nonadiabatic quantum motion in Fig. 1b.
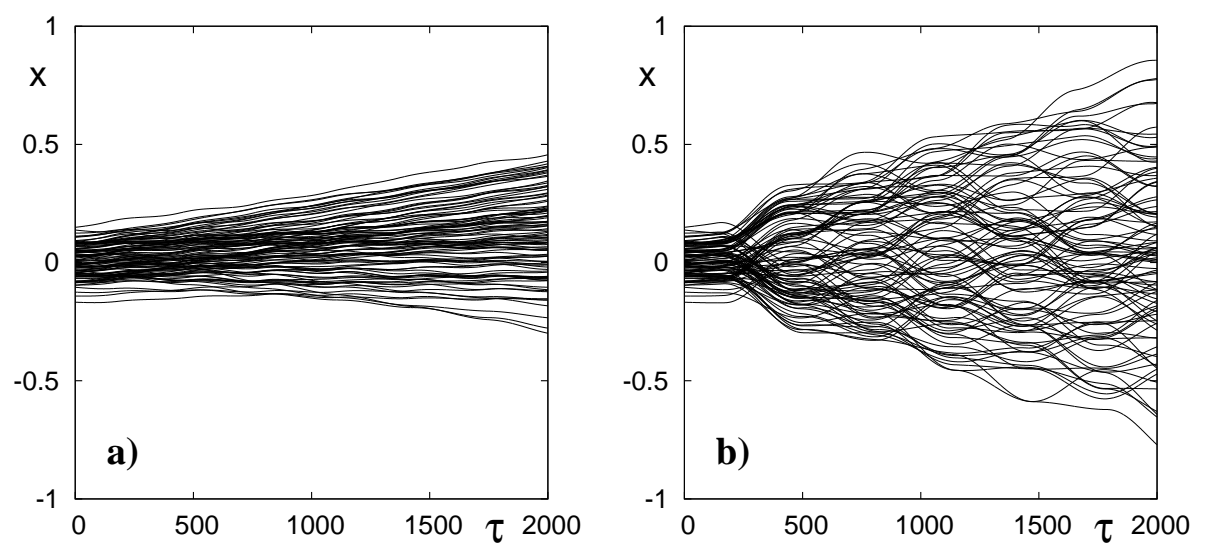

Figure 6: Classical atomic trajectories in the moving frame of reference. (a) Regular bundle $(\Delta=$ $0.3)$ corresponding to adiabatic quantum motion in Fig. 2a and (b) weakly chaotic bundle $(\Delta=0.1)$ corresponding to nonadiabatic quantum motion in Fig. 2b. 